\title{
Using reverse microdialysis to simulate and explore root exudation 'hot spots' in the soil
}

\author{
CHRISTINA KAISER ${ }^{1}$, JULIA WIESENBAUER ${ }^{1}$, STEFAN \\ GORKA $^{1}$, ALEXANDER KOENIG ${ }^{1}$, LILIAN MARCHAND ${ }^{1}$ \\ AND ERICH INSELSBACHER ${ }^{2}$ \\ ${ }^{1}$ University of Vienna \\ ${ }^{2}$ University of Natural Resources and Life Sciences \\ Presenting Author: christina.kaiser@univie.ac.at
}

Plant roots exude a variety of low-molecular weight compounds, such as sugars, amino acids or organic acids into the soil with significant effects on physico-chemical soil processes and microbial activities. Soil microbes use these compounds as a source of C and energy, leading to the formation of 'hot spots' of high microbial activity and growth. Investigating microbial and physico-chemical processes at such exudation hot spots, however, has remained a challenge, as small and distinct spots in the soil are difficult to access by experimental observation.

Here, we used reverse microdialysis as a novel tool to investigate processes in the immediate surrounding of exudation hotspots. This technique allows to introduce chemical compounds at distinct, small spots in undisturbed soil via microdiffusion, i.e. simulating a 'root exudation event'. Soil metabolites can subsequently be collected from the same spots at a high temporal resolution. We combined this approach with ${ }^{13} \mathrm{C}$ stable isotope tracing to quantify microbial respiration of the added compounds as well as their incorporation into microbial biomass.

Our results show that the punctual release of a mixture of sugars and organic acids over a few hours significantly changed the temporal dynamics of certain other soil metabolites (i.e. acetate, propionate, ammonium and nitrate) right at this spot over the following days. The rise of certain metabolites indicates that microbial metabolism has switched from aerobic to anaerobic. This could be explained by the sudden peak in microbial respiration triggered by the added $\mathrm{C}$ compounds, which may have depleted the spot in oxygen. Long-term consequences of the exudation event were still visible 12 days later in altered metabolite abundances.

Root exudation spots, are, as roots are constantly growing and mainly exuding from their tip, heterogeneously and dynamically distributed in soil. Our results show that each of these flickering exudation spots likely significantly alter microbial metabolic processes and thereby the chemical composition in its immediate vicinity for a comparable long time. Plant root exudation may therefore facilitate the temporal dynamic and spatial diversity of the soil at the microscale, with potential implications for biogeochemical cycling. 